\title{
Économie de l'Afrique contemporaine
}

Pour les sciences sociales, le chiffre est une donnée à la fois fascinante et ambiguë. Sa validité est souvent fragile, toujours susceptible d'être remise en cause, mais son pouvoir de suggestion, grâce à sa précision réelle ou apparente, est considérable. Sa capacité à mettre en ordre le monde dépasse ainsi largement le cadre scientifique pour constituer un outil politique essentiel des sociétés contemporaines, comme l'a montré Alain Desrosières. Son universalisation est directement liée à la connexion de plus en plus étroite des différentes parties du monde à partir de l'époque moderne, puis au développement d'institutions internationales depuis la Seconde Guerre mondiale. Pour autant, la production du chiffre reste une question ouverte, de même que sa capacité à décrire de manière fiable des réalités sociales et économiques. C'est encore plus vrai lorsqu'il est appliqué d'une manière standard à un contexte profondément différent de celui qui lui a donné naissance.

Cette difficulté trouve une nouvelle actualité grâce aux deux livres incisifs et provocants de Morten Jerven consacrés à la production de données quantitatives et à l'usage du chiffre dans les recherches portant sur l'économie africaine, actuelle et ancienne. L'objectif de ses travaux est de démontrer la faiblesse de beaucoup des statistiques utilisées par les économistes du développement. C'est en particulier le cas du produit intérieur brut (PIB) dans les pays d'Afrique subsaharienne anglophone depuis les indépendances (Botswana, Ghana, Kenya, Malawi, Nigeria, Tanะanie, Ouganda, Zambie), que M. Jerven étudie longuement pour en démonter les principes de fabrication très discutables. Le calcul du PIB agrégeant des données comptables de nature très diverse, sa mesure constitue une opération délicate et sujette à caution, y compris dans les pays développés qui possèdent des instituts producteurs de statistiques performants et peuvent s'appuyer sur une tradition bien ancrée. La difficulté est encore plus grande en Afrique où les réalités économiques et sociales sont très hétérogènes et 
juxtaposent, plus qu'ailleurs, des phénomènes monétaires et non monétaires, échappant de ce fait au calcul et à la mise en nombre des économistes. Les cas récents de forts réajustements du PIB de certains pays africains, notamment le Ghana, à la suite d'un changement dans la méthode de calcul, conduisant à une appréciation très différente de thèmes essentiels comme la validité des politiques économiques mises en œuvre ou la stagnation des économies africaines, sont matière à réflexion pour M. Jerven quant à la grande fragilité des savoirs quantitatifs. Selon lui, l'attention croissante portée à un récit global sur le continent construit des fictions fondées sur des chiffres douteux et hétérogènes, compromettant considérablement la possibilité d'interpréter correctement les économies africaines. Il plaide pour un changement méthodologique, un retour à l'échelle nationale et une meilleure historicisation, localisation et contextualisation de la production des données chiffrées.

Les travaux de M. Jerven ouvrent sur une pluralité de questions abordées par les trois contributions réunies dans ce dossier. Suggérons en trois. La première relève de la sociologie de la quantification. Qui sont les producteurs de statistiques? Quelles sont les institutions, publiques ou privées, qui organisent, financent et exploitent la récolte des chiffres? Cette interrogation doit être très large, incluant aussi bien le niveau national, assez mal étudié, qu'international, tant il est vrai que beaucoup de données sont produites par des experts et des organismes, la Banque mondiale en particulier, qui ne travaillent pas dans les pays dont ils retracent les comptabilités.

La seconde question, sise au cœur des contributions d'Agnès Labrousse et de Boris Samuel, porte sur le type d'incertitude qui entoure la validité des données chiffrées, thème sur lequel les ambiguïtés de M. Jerven sont à la fois déconcertantes et heuristiques. Doit-on privilégier une perspective réaliste et considérer qu'il est possible d'obtenir une valeur approchée des agrégats - à commencer par le PIB - que l'on entend estimer? Les difficultés de mesure relèveraient dès lors de la médiocrité des institutions statistiques africaines ainsi que des administrations nationales, lesquelles sont peu capables de produire et d'enregistrer des statistiques de nature économique, le relais étant pris par les institutions internationales, ellesmêmes productrices de données chiffrées dont l'origine n'est pas moins incertaine. L'effort prioritaire, dans cette perspective, devrait être d'élaborer de meilleurs instruments de mesure. Doit-on au contraire s'interroger sur ce que l'on cherche à mesurer et sur l'adéquation entre les statistiques produites et les réalités économiques? Depuis les travaux d'A. Desrosières, largement mobilisés par A. Labrousse et B. Samuel, on sait que toute mesure chiffrée est une convention dont il importe de bien définir le contenu et les contours pour savoir ce que l'on saisit. La notion de PIB, pour incomplète qu'elle soit, ne peut avoir la même signification pour des pays comme la France ou les États-Unis, où l'économie est structurée autour des échanges marchands, et des pays africains où les activités de production et de consommation prennent des formes plus variées, au sein desquelles les échanges d'équivalents mesurables (et taxables par l'État) ne sont pas toujours dominants. Le problème n'est pas seulement de pouvoir mesurer mais de savoir ce que l'on mesure, connaissance qui bien souvent requiert d'autres expertises que celles de l'économiste, celles de l'anthropologue, par exemple, ou de l'historien. Ainsi, les travaux de Vincent Bonnecase ont attiré notre attention sur la nécessité de penser les chiffres coloniaux en questionnant la manière dont ils ont été produits, mais aussi en tenant compte de leurs significations dans leur contexte de fabrication, afin de ne pas leur accorder une fonction et un sens qu'ils n'avaient pas au moment de leur élaboration. 
La troisième question, posée dans la contribution de Denis Cogneau, porte sur la possibilité d'analyser les économies africaines et leur croissance, ancienne et actuelle, en usant de ces données quantitatives peu fiables et mal construites. Poser cette question est d'autant plus nécessaire que les économistes ont eu tendance au cours des dernières années à abandonner les études monographiques, cadre qui permet de mieux cerner la validité des estimations chiffrées, au profit des études internationales appuyées sur des méthodes économétriques, grandes consommatrices de données agrégées. Ces dernières ouvrent sur des comparaisons entre pays, mais au prix d'un grand aveuglement quant au contenu réel des données mobilisées et à leur commensurabilité. L'histoire économique dans la longue durée relève en partie de difficultés similaires, estime M. Jerven, puisque les grands récits proposés aujourd'hui par les économistes sur le mal-développement de l'Afrique et les multiples divergences historiques qui éloigneraient le continent des principes d'une bonne économie de marché souffrent des mêmes insuffisances, à savoir un manque d'identification et de contenu de beaucoup des données utilisées et des comparaisons entre pays fondées sur de fragiles estimations économétriques. Néanmoins, ni les fictions bureaucratiques, ni les doutes sur les données utilisées pour fabriquer le PIB, ni l'existence d'une économie politique du chiffre et l'instrumentalisation de ceux-ci à des fins politiques, ne sont des problématiques exclusivement africaines. Beaucoup des questions posées dans ce dossier dépassent le continent africain.

Ainsi, les discussions des travaux de M. Jerven se situent au cœur du projet qui anime les Annales depuis leur fondation. Elles permettent, comme la revue l'avait fait à l'occasion de la parution du livre de Thomas Piketty, Le capital au XXI ${ }^{\mathrm{e}}$ siècle, de confronter les méthodes et les outils de l'économie avec celles des autres disciplines que sont l'histoire, la sociologie et l'anthropologie ${ }^{1}$. Ces discussions donnent également l'occasion de réinscrire les usages sociaux et politiques des chiffres produits par ces outils de mesure dans la perspective d'une critique épistémologique. De l'un à l'autre, les sciences sociales peuvent faire la démonstration de leur contribution à la réflexion collective sur les politiques publiques sans pour autant renoncer à leur autonomie.

Les Annales

1 - Dossier «Lire Le capital de Thomas Piketty », Annales HSS, 70-1, 2015, p. 5-138. 